\title{
A Statistical Representation of Stacking Disorder in Layered Covalent Organic Frameworks
}

Yingying Zhang ${ }^{\dagger}$, Miroslav Položij ${ }^{\dagger^{*}}$, Thomas Heine ${ }^{\dagger, t, z^{*}}$

${ }^{\dagger}$ Faculty of Chemistry and Food Chemistry, TU Dresden, Bergstrasse 66c, 01069 Dresden, Germany

${ }^{*}$ Helmholtz-Zentrum Dresden-Rossendorf, Institute of Resource Ecology, Leipzig Research Branch, Permoser Str. 15, 04316 Leipzig

${ }^{z}$ Department of Chemistry, Yonsei University, Seodaemun-gu, Seoul 120-749, Korea

*Email: thomas.heine@tu-dresden.de

miroslav.polozij@tu-dresden.de

\begin{abstract}
Covalent organic frameworks (COFs) are among the fastest growing classes of materials with an almost unlimited number of achievable structures, topologies, and functionalities. The exact structure of layered COFs is, however, hard to determine due to often significant mismatch between experimental powder X-ray diffraction pattern (PXRD) and predicted geometries. We attribute these discrepancies to an inherent disorder in the stacking of layered COFs, invalidating
\end{abstract}


standard theoretical 3D models. We have represented structure of COF-1, COF-5 and ZnPc-pz by stacking layers following the Maxwell-Boltzmann energy distribution of their stacking modes. Simulated PXRD patterns of the statistical COF models are close to the experiment, featuring an agreement in peak intensity, width and asymmetry never obtained before. The rarely considered $\mathrm{ABC}$ stacking mode proved to be important in layered $\mathrm{COFs}$, as well as including solvent molecules. Our model also shows several general features in PXRD originating from the stacking disorder.

\section{INTRODUCTION}

Covalent organic frameworks (COFs) are crystalline, porous networks built from organic linkers via covalent bond formation. ${ }^{1-3}$ Most COFs to date are layered materials composed of atomically thin two-dimensional (2D) sheets, which create stacked crystals via non-bonding interactions. ${ }^{2,4,5}$ Commonly, these materials are referred as $2 \mathrm{D} \mathrm{COFs,} \mathrm{a} \mathrm{somewhat} \mathrm{misleading} \mathrm{nomenclature} \mathrm{as}$ most representatives are layered 3D bulk materials. Although we will show that in most layered COFs there is no periodicity normal to the $2 \mathrm{D}$ crystal plane and thus a common definition of a $2 \mathrm{D}$ periodic material (translational symmetry only in two dimensions) fits, we will, following the traditional nomenclature of related layered materials, ${ }^{6}$ employ here the term layered COFs (LCOFs).

Although some COF structures can be precisely identified by single crystal X-Ray diffraction ${ }^{7,8}$. However, the great challenge in the synthesis of the COF single crystal, especially LCOFs, renders the revealing of most of the COFs crystal structure still heavily depends on the powder XRD together with the theoretical simulation. There is no unambiguous knowledge of the atomistic LCOF structures as powder X-ray diffraction (PXRD) data itself does not contain sufficient 
information to resolve the structure in atomistic resolution, and predicted structures do not closely match experimental PXRD patterns. LCOF PXRD patterns commonly have signs of structural disorder with wide, diffused peaks, while those simulated from predicted structures feature many narrow peaks (Figure 1). ${ }^{9-11}$ Recently, a total scattering study by Pütz et al. has shown that LCOFs with a well-defined local structure exhibit a long-range stacking disorder. ${ }^{12}$ This disorder systematically causes false interpretation of experimental results, which commonly is mapped to an average structure of high symmetry in stacking direction. Similar disorder was identified layered zeolites ${ }^{13}$ and molecular crystals. ${ }^{14}$

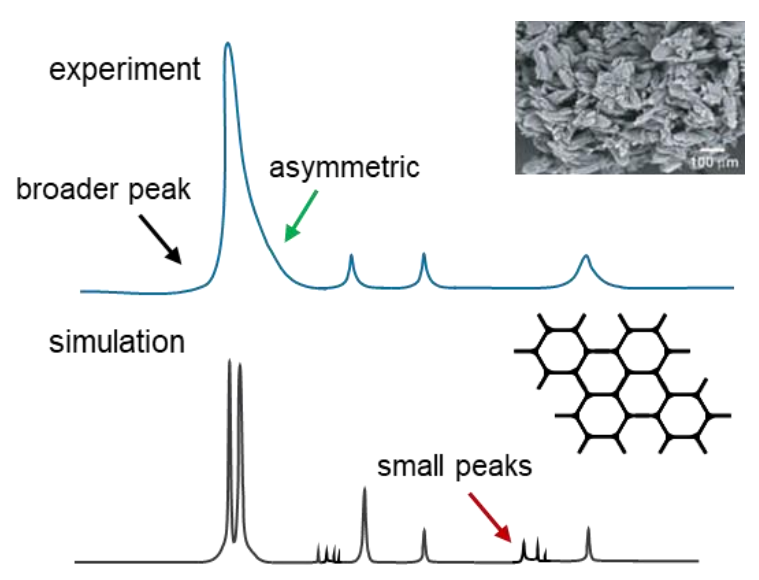

Figure 1. A sketch of common differences of experimental and theoretical PXRD patterns in LCOFs.

Here, we present a general approach to simulate the crystal disorder in LCOFs, by statistically stacking COF monolayers in a sequence following a Maxwell-Boltzmann distribution of their relative stacking energies. This model yields unprecedented agreement of PXRD patterns between experiment and theory, to the point of being able to identify the actual ratios of stacking modes present in the samples. 


\section{RESULTS AND DISCUSSION}

LCOF stacking. We have chosen the two most common LCOF framework lattice types; namely honeycomb $(\mathrm{hcb})$ (more correctly kagome $(\mathrm{kgm})$, for simplicity we refer to the building block centers which form $h c b$ lattices) represented by COF-1 and COF-5, and square (sql) lattices,

represented by ZnPc-pz. ${ }^{10,15}$ These LCOFs have flat, atom-thin layers with only ZnPc-pz featuring bulky tert-butyl side groups reaching out of the layer plane (Figure 2e).

A search for all possible stacking modes in the three LCOFs using bulk structural models employing the Density-Functional based Tight-Binding (DFTB) method resulted in five distinct types (Figure 2, Tables 1, SI-2, 3):

i. $\quad \mathbf{A A},(h c b$ and $s q l)$; with layers directly above each other. The "eclipsed" stacking mode (AAe) is energetically highly improbable, so the layers shift laterally by about $1.5 \AA$ to AAs. This shift is confirmed theoretically and experimentally. ${ }^{12}$

ii. $\quad \mathbf{A B}(h c b)$; with top layer shifted laterally to middle of the hexagonal pore, i.e. by $[1 / 3,1 / 3]$ unit cell (UC) and $[-1 / 3,-1 / 3]$ in the next layer. $\mathbf{A} \mathbf{B}_{\mathbf{e}}$ and $\mathbf{A} \mathbf{B}_{\mathbf{s}}$ (same shift types as $\mathrm{AA}_{\mathbf{s}}$ ) are almost isoenergetic and can be of low energy.

iii. $\quad \mathbf{A B C}(h c b)$; layers shifted laterally by $[1 / 3,1 / 3]$ UC as in $\mathrm{AB}$ and again $[1 / 3,1 / 3]$ in the next layer. The interlayer shift is identical to $\mathrm{AB}_{\mathrm{s}}$, but a different superlattice appears in projection along layer normal. $\mathrm{ABC}$ is known in other $h c b$ COFs. ${ }^{16,17}$ Due to layer corrugation (Figure SI-3) it is the most stable structure for COF-1 and COF-5.

iv. $\quad \mathbf{A B}_{\text {s-diag }}(s q l)$; layers shifted laterally by $[0.5,0.5]$ UC. Only structures with small additional shift $\mathrm{AB}_{\mathrm{s}-\mathrm{diag}}$ exist in $\mathrm{ZnPc-pz}$, all of them high in energy. 
v. $\quad \mathbf{A B}_{\text {s-axis }}(s q l)$; layers shifted laterally by $[0.5,0]=[0,0.5] \mathrm{UC}$. The shift changes to $\left[ \pm^{1 / 3}, 0\right]$ or $[0, \pm 1 / 3]$ for $\mathrm{ZnPc}$-pz due to the bulky side chains $\left(\mathbf{A B C}_{\mathrm{s} \text {-axis }}\right)$. The most stable structure of ZnPc-pz.

Table 1. Relative energies per building block (BB) of selected stacking modes of COF-1, COF-5 with and without solvent molecule and ZnPc-pz. For overview of all investigated structures see Table SI-5.

\begin{tabular}{ccccc|cc}
\hline $\begin{array}{c}\text { PBE }+ \\
\text { D3(BJ)/DZP }\end{array}$ & COF-1 & $\begin{array}{c}\text { Erel: kJ/mol/BB } \\
\text { mesi }\end{array}$ & COF-5 & $\begin{array}{c}\text { COF-5 } \\
+ \text { mesi }\end{array}$ & PBE + & $\begin{array}{c}\text { Erel: } \\
\mathrm{kJ} / \mathrm{mol} / \mathrm{BB}\end{array}$ \\
\hline $\mathrm{AA}_{\mathrm{e}}$ & 35.5 & - & 49.1 & 54.0 & $\mathrm{AA}_{\mathrm{s}}$ & ZnPc-pz \\
$\mathrm{AA}_{\mathrm{s} 1}$ & 7.5 & 44.3 & 0.1 & 0.0 & & 42.4 \\
$\mathrm{AB}_{\mathrm{e}}$ & 14.5 & 0.0 & 104.7 & 56.5 & $\mathrm{AB}_{\text {s-diag }}$ & 220.1 \\
$\mathrm{AB}_{\mathrm{s} 1}$ & 16.4 & 14.7 & 98.6 & 51.1 & & \\
$\mathrm{ABC}_{\mathrm{e}}$ & 0.8 & 0.0 & - & - & $\mathrm{ABC}_{\text {s-axis }}$ & 0.0 \\
$\mathrm{ABC}_{\mathrm{s}}$ & 0.0 & 21.0 & 0.0 & 33.1 & & \\
\hline
\end{tabular}

Due to the large discrepancy between PXRD patterns of as-synthesized and activated COF-1 and COF-5, we have modeled the presence of solvent by adding mesitylene molecules ("+mesi"), considering one mesitylene molecule per pore, the same number as the original experimental paper (= full coverage in $\mathrm{ABC}$, Figure 4$).{ }^{10}$ The solvent changes the $\mathrm{ABC}_{\mathrm{e} / \mathrm{s}}$ structure, preventing layer corrugation. This affects stacking energetics considerably; in $\mathrm{COF}-1, \mathrm{ABC}_{\mathrm{e}}$ and $\mathrm{AB}_{\mathrm{e}}$ become isoenergetic, while being more stable than $\mathrm{AA}_{\mathrm{s}}$, whereas in COF-5 $\mathrm{AA}_{\mathrm{s}}$ is preferred (Table 1). 
Statistical COF Models. The presence of interlayer shifts breaks the LCOF symmetry, so the interlayer shifts split into ensembles of isoenergetic non-equivalent variants. The two shift directions in COF-1 and COF-5 result in 6 and 12 shifts, respectively, and in ZnPc-pz there are two pairs of shifts (Figure 2d). Thus, due to configurational entropy, any LCOF must have a disordered structure with statistical set of these shift directions (Figure SI-5).

(a)

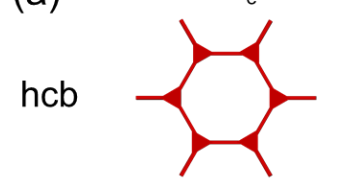

(b)

hcb

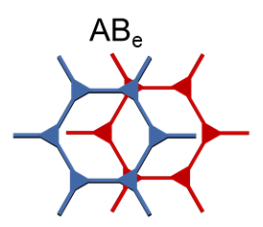

$\rightleftarrows \frac{1}{2}$

(c)

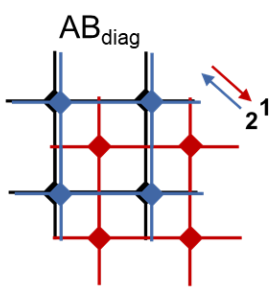

sql
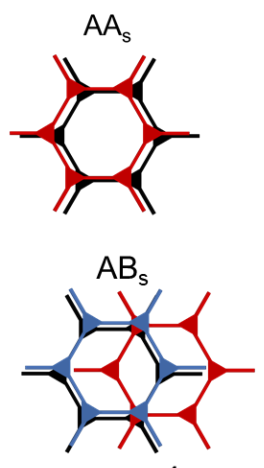

$\rightleftarrows \frac{1}{2}$

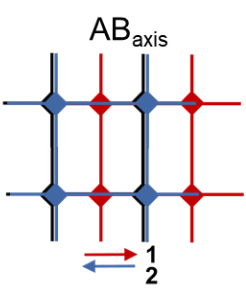

(e)
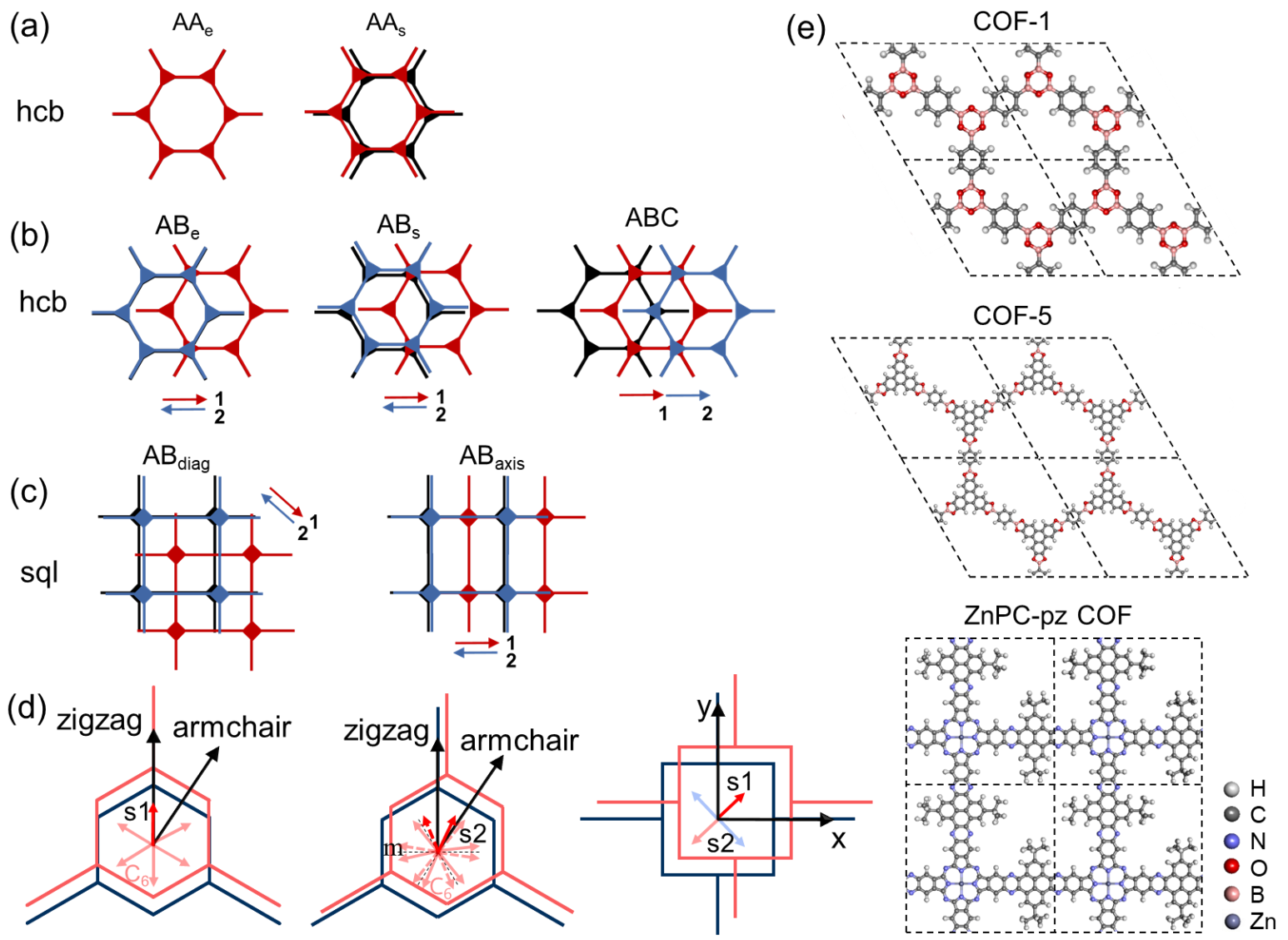

Figure 2. (a) $\mathrm{AA}_{\mathrm{e} / \mathrm{s}}$ stacking modes of $h c b$, sql lattices. (b) $\mathrm{AB}_{\mathrm{e} / \mathrm{s}}$ and $\mathrm{ABC}_{\mathrm{e}}$ in $h c b$, (c) $\mathrm{AB}_{\text {diag }}$ and $\mathrm{ABC}_{\text {axis }}$ in $s q l$ lattices. Successive layers in black, red and blue. (d) Possible small shift-vectors in $h c b$, and $s q l$. (e) Atomic structure of COF-1, COF-5 and ZnPc-pz.

To obtain realistic LCOF structure models, we first create a set of all possible interlayer shifts using Maxwell-Boltzmann distribution of their relative energies (at the synthesis temperature ${ }^{10,15}$ ). 
We then construct the bulk structures layer-by-layer, using Markov chain random walk algorithm with different stacking modes. We have verified the long-range shift direction independence optimizing all COF-1 trilayer $\mathrm{AA}_{\mathrm{s}}$ structures, (Table SI-6). The resulting structures are denoted $\mathrm{AA} / \mathrm{AB} / \mathrm{ABC}^{\text {stat }}$.

Generally, energetics allows combining multiple stacking types $\left(\mathrm{AA}_{\mathrm{s}}, \mathrm{ABC}_{\mathrm{e} / \mathrm{s}}, \ldots\right)$ in the same crystal. We denote such combinations $\mathrm{AA}_{\mathrm{s}}: \mathrm{AB}(\mathrm{C})^{\text {stat }} \mathrm{X}: \mathrm{Y}$, specifying their proportion from statistical distribution. In the special case for $\mathrm{AB}_{\mathrm{e} / \mathrm{s}}$ and $\mathrm{ABC}_{\mathrm{e} / \mathrm{s}}$, which have the same interlayer shift, we denote them $\mathrm{AB}(\mathrm{C})^{\text {stat }}$.

\section{COF-1, COF-5 and ZnPc-pz structures and their PXRD patterns}

We have verified our model by simulating PXRD patterns and comparing them with experiment (Figure 3, peak positions Table SI-7). The statistical distribution of stacking modes yields PXRD patterns similar to experiment, both in position and shape, in contrast to the sharp peak distribution obtained from homogeneous stacking as obtained from minimal unit cells (Figure 3, Figures SI-9 to SI-11). Our statistical method "smoothens" the predicted patterns, in agreement with the findings of Pütz et al. for $\mathrm{AA}_{\mathrm{s}}$ TTI-COF (see also Figure SI-12). ${ }^{12}$

The PXRD patterns of the disordered crystals show several common features. In both $h c b$ and $s q l \mathrm{LCOFs}$, the $\mathrm{AA}^{\text {stat }}$ structures have a pronounced main peak in the range of $2 \theta=4^{\circ} \ldots 9^{\circ}$. Only $h c b \mathrm{AB}^{\text {stat }}$ and $s q l \mathrm{AB}(\mathrm{C})_{\mathrm{s} \text {-axis }}^{\text {stat }}$ patterns feature this peak too, though, whereas $h c b \mathrm{AB}(\mathrm{C})^{\text {stat }}$ and sql $\mathrm{AB}_{\mathrm{s}-\text { diag }}^{\text {stat }}$ have only low-intensity peak in this range. Both these structures feature a new superlattice motif forming in projection along the layer normal, causing the distinct pattern (Figure SI$2,13)$. 
(a)

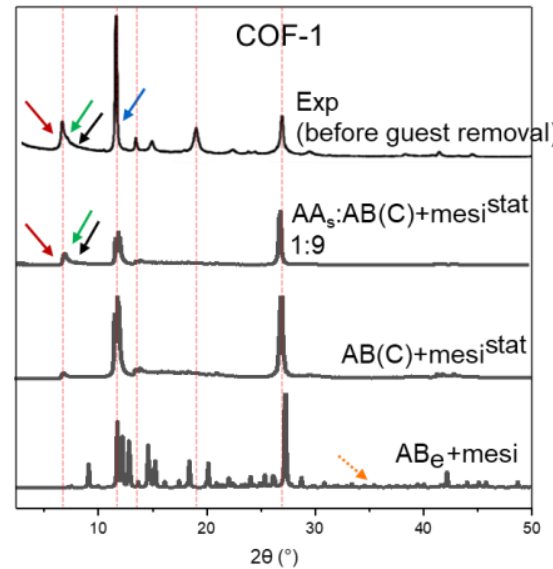

(c)

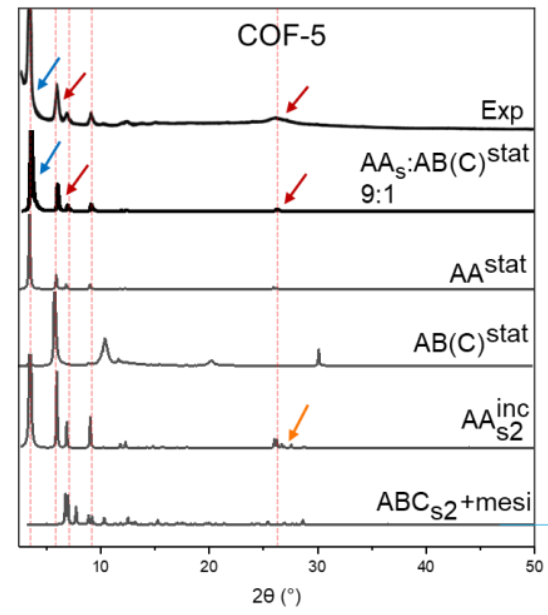

(b)

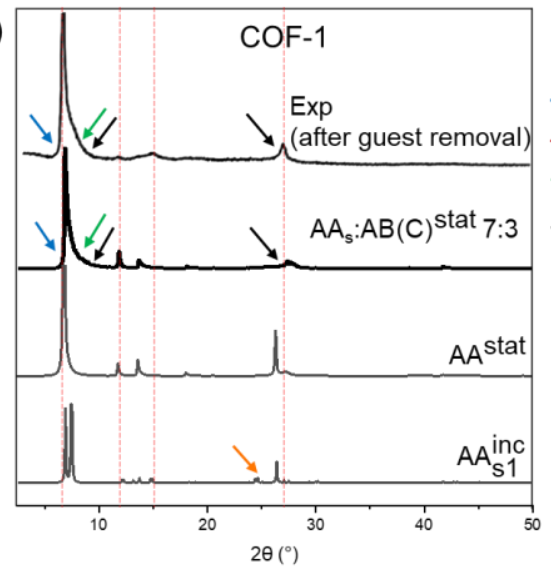

(d)

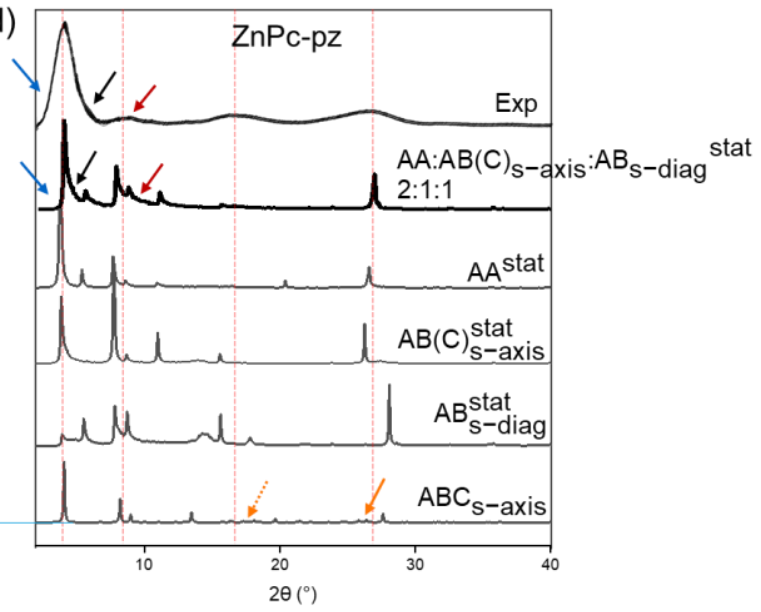

Features in statistical model:

$\longrightarrow$ High intensity

$\longrightarrow$ Low intensity

$\longrightarrow$ Asymmetric

Features disappeared in statistical model:

$\ldots . . . . . \rightarrow$ Fine structure

$\longrightarrow$ Small peaks

Figure 3. Comparison of experimental and simulated PXRD patterns of a) as synthetized COF-1, b) evacuated COF-1, c) COF-5 and d) ZnPc-pz COF. Each section depicts PXRD patterns of the experimental sample (top, from refs. ${ }^{10,15}$ ), various statistical models (middle) and minimal model without disorder (bottom). Arrows highlight major characteristic features.

After adding mesitylene (solvent) molecules in the simulation, the statistical model predicts an occurrence of $92 \%$ of the $\mathrm{ABC}_{\mathrm{e}}$ stacking mode for as-synthesized COF-1 (Table 1). The resulting PXRD pattern, $\mathrm{AA}_{s}: \mathrm{AB}(\mathrm{C})+$ mesi $^{\text {stat }}$ 1:9 (Figure 3a), matches the experiment well, with a lowintensity peak at $6-8^{\circ}$, higher intensity peak at $11-13^{\circ}$ and an intensive peak at $27^{\circ}$. Removal of mesitylene molecules shifts the last peak position to higher angles (Figure 4) due to layer corrugation. 
The COF-1 experimental PXRD pattern changes considerably after solvent evacuation (Figure $3 b)$. The $6-10^{\circ}$ peak becomes the most intensive one and a wide peak forms around $2 \theta=27^{\circ}$. This matches well our simulated AA ${ }^{\text {stat }}$ PXRD pattern, which only lacks asymmetry of the main peak. Thus, $\mathrm{COF}-1$ transforms from $\mathrm{ABC}_{\mathrm{e} / \mathrm{s}}$ to $\mathrm{AA}_{\mathrm{s}}$ stacking during solvent removal, likely because that the needed 1D pores for solvent diffusion are lacking in $\mathrm{ABC}_{\mathrm{e} / \mathrm{s}}$ stacked COF-1 (Figure SI-3).

Stacking change due to evacuation is inherently a kinetic process, so the resulting stacking order will not follow Maxwell-Boltzmann statistics. We have therefore sampled $\mathrm{AA}_{\mathrm{s}}: \mathrm{AB}(\mathrm{C})+$ mesi $^{\text {stat }}$ structures with varying ratios (Figure 5), assuming that all $\mathrm{AB}(\mathrm{C})^{\text {stat }}$ layers still contain mesitylene. The $6-8^{\circ}$ peak intensity decreases together with the $\mathrm{AA}_{\mathrm{s}}$ probability. This peak also becomes asymmetric towards higher $2 \theta$ angles when $\mathrm{ABC}_{\mathrm{e} / \mathrm{s}}$ content exceeds $25 \%$. This asymmetry is also seen in the experimental PXRD pattern, with best agreement in 3:7 $\mathrm{AA}_{\mathrm{s}}: \mathrm{AB}(\mathrm{C})+\mathrm{mesi}^{\text {stat }}$ structure. Both $\mathrm{AA}^{\text {stat }}$ and $\mathrm{AB}(\mathrm{C})^{\text {stat }}$ modes are present in the same crystals, as the peak asymmetry disappears, if the same proportion of isolated $\mathrm{AA}^{\text {stat }}$ and $\mathrm{AB}(\mathrm{C})^{\text {stat }}$ crystallites is used (Figure SI$14)$.
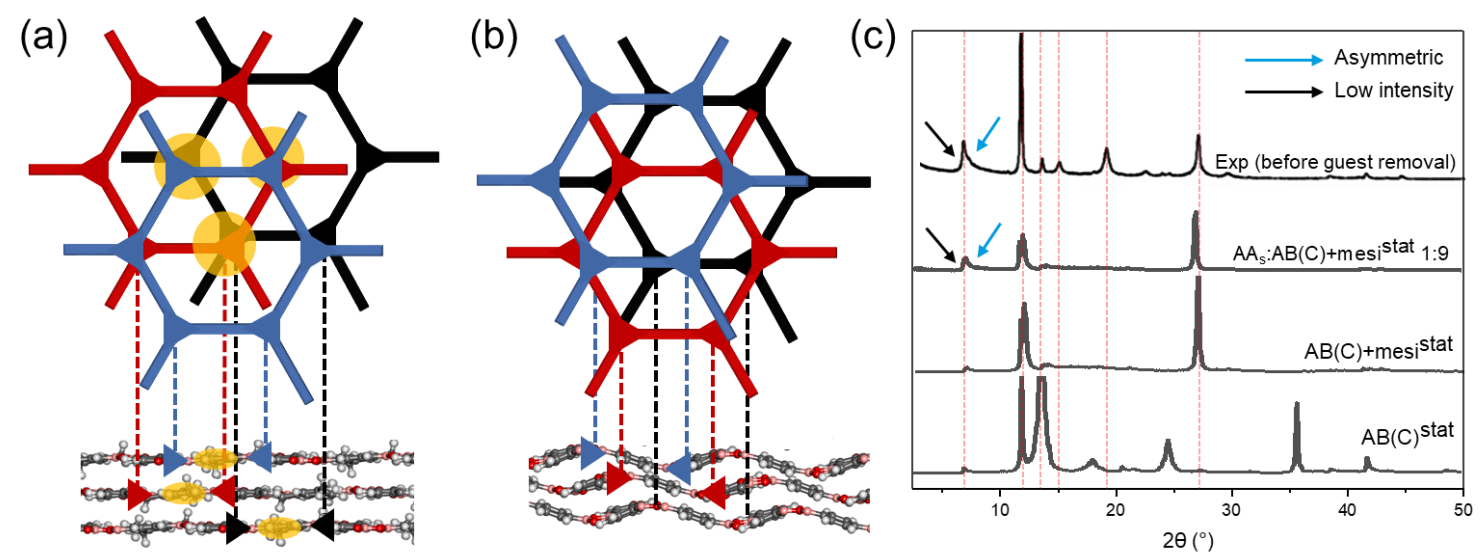

Figure 4. ABC COF-1 structure (a) with pores filled with mesitylene, (b) without any guest molecules; (c) $\mathrm{AB}(\mathrm{C})^{\text {stat }}$ and $\mathrm{AB}(\mathrm{C})+$ mes $^{\text {stat }}$ PXRD patterns compared with the best fitting mixed $\mathrm{AA}_{\mathrm{s}}: \mathrm{AB}(\mathrm{C})+\mathrm{mesi}^{\text {stat }}$ structure and experimental PXRD pattern from ref ${ }^{10}$. 


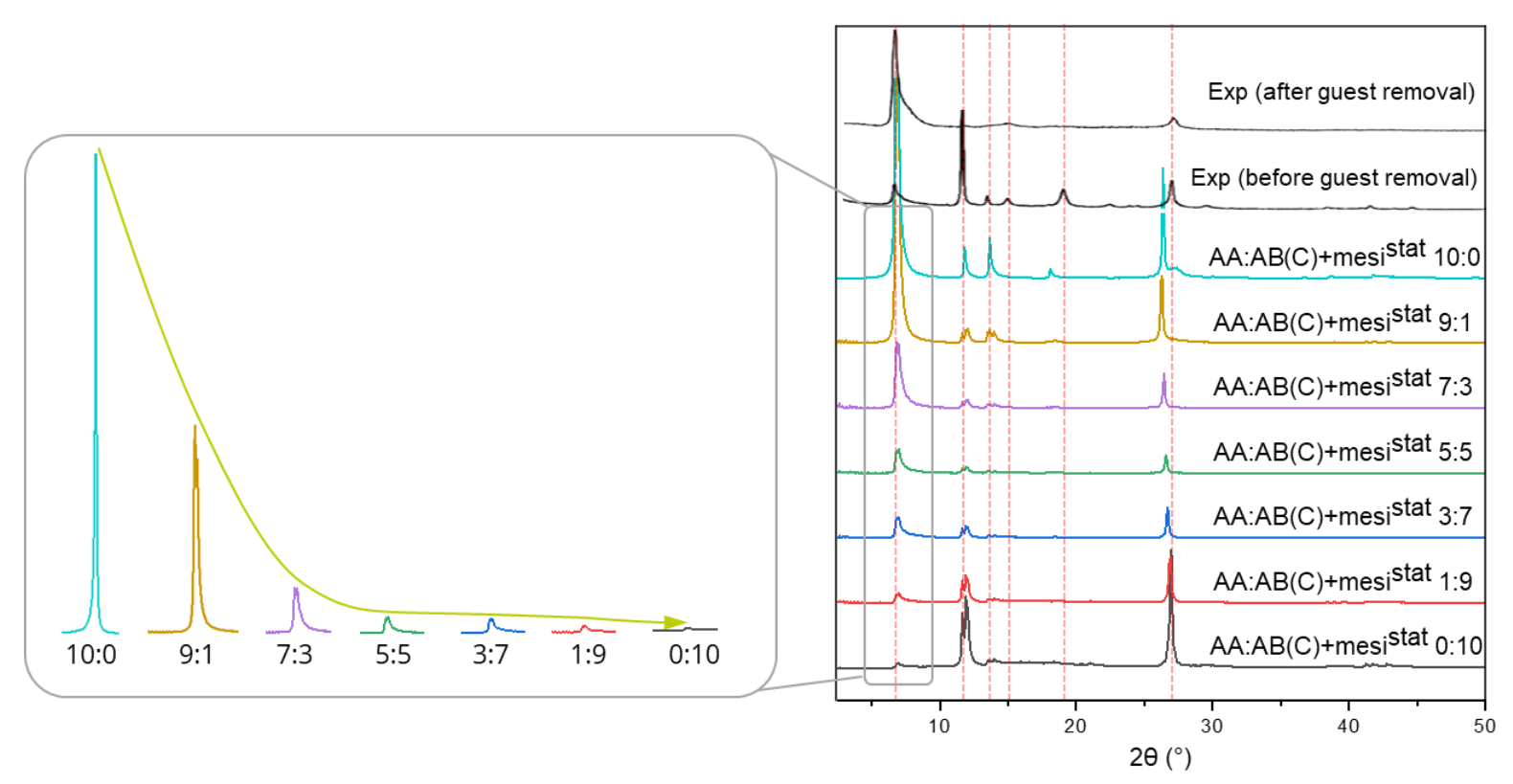

Figure 5. PXRD patterns of COF-1 $A A_{s}: A B(C)+$ mesi $^{\text {stat }}$ model with varying ratios. The top two patterns (black) are experimental result from ref. ${ }^{10}$. Left figure compares peaks between 5-9 $2 \theta$.

The most probable stacking mode of COF-5 is $\mathrm{AA}_{\mathrm{s}}$ (Table 1). The corresponding $\mathrm{AA}^{\text {stat }}$ pattern directly matches the experimental pattern (Figure $3 \mathrm{c}$ ). The wide peak around $2 \theta=27^{\circ}$ observed in experiment suggests there is some structural disorder. This can originate from a small proportion $(<10 \%)$ of $\mathrm{AB}(\mathrm{C})^{\text {stat }}$ (Figure SI-16).

ZnPc-pz energetic profile favors the $\mathrm{ABC}_{\text {s-axis }}$ structure (Table 1), and the PXRD patterns suggest either AAstat or $\mathrm{AB}(\mathrm{C})_{\text {axis }}^{\text {stat }}$ stacking mode due to high intensity of $4^{\circ}$ peak. The experimental powder pattern shows low crystallinity, though, so we have also sampled the stacking mode mixing as a potential disorder source. The combination of three main stacking modes (Figure SI-17) shows features seen in both $h c b$ LCOFs, i.e. low-intensity of $\mathrm{AB}_{\text {diag }}^{\text {stat }} 4^{\circ}$ peak and asymmetrical peaks forming in mixed-type crystals. This suggests that the peak asymmetry is in general a distinguishing feature for reading LCOF PXRD data. 
Beyond the materials mentioned above, we have applied the statistical method to other LCOFs using already published energetic and structural data (Figure SI-18). ${ }^{18,19}$ The results show an improvement of the match of experimental and predicted PXRD when using the statistical model. Furthermore, the results again hint a possibility of mixing of several stacking modes in the experimental samples, producing the same features observed in the detailed COF-1 and ZnPc-pz investigation.

Electronic Structure of disordered LCOF. Electronic structure in LCOFs is sensitive to the layer stacking order. For example, it was shown for COF-5 that different small-UC models, $\mathrm{AA}_{\mathrm{s}}^{\mathrm{inc}}$, $\mathrm{AA}_{\mathrm{s}}^{\mathrm{serr}}$ yield electronic structures of significant differences in band gap and dispersion. ${ }^{20}$ Therefore, we have evaluated the electronic structure of COF-1 and COF-5 in statistical stacking order. As a compromise between good statistics and computational costs, we have built multilayered bulk "snapshots" of disordered LCOF structure. Due to the low symmetry the band structures become crowded, so we focus on the densities-of-states (DOS) differences.

The $\mathrm{AA}_{\mathrm{s}}^{\mathrm{serr}}$ model of both COF-1 and COF-5 yields larger bandgaps than corresponding inclined structures (Figure 6, Figure SI-1). The AA ${ }^{\text {stat }}$ bandgap is close to that of serrated structure, or even higher, although it also depends on the local structure (Figure SI-19). A sharp peak in DOS forms at the edge of both valence and conduction bands of the statistical structure. The solvent-imposed structure change has severe consequences for the electronic structure, which has more diffuse bands and narrower bandgap (Figure SI-20, 21). 

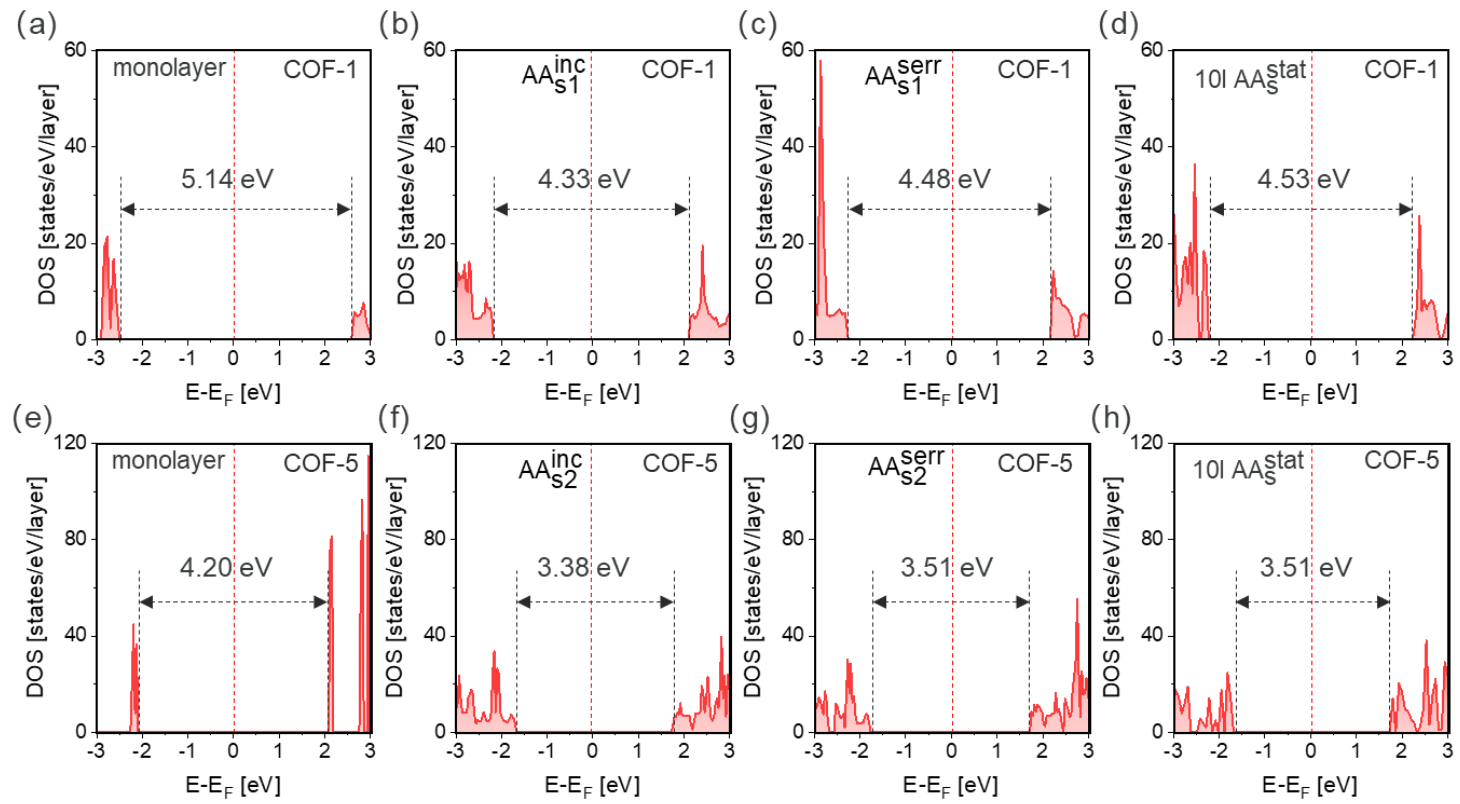

Figure 6. Densities of states of (a), (e) monolayer, (b), (f) inclined, and (c), (g) serrated $\mathrm{AA}_{\mathrm{s} 1}$ bulk structures with bilayers per UC, compared with (d), (h) 10-layer AA ${ }^{\text {stat }}$ model for COF-1 and COF5. The red dashed line denotes the Fermi level.

\section{CONCLUSIONS}

We have shown that the layered COFs, despite having crystalline layers, exhibit disorder along third axis. Statistical stacking order thus needs to be considered to properly describe their structure and properties. We have developed a procedure to create atomistic models of COF-1, COF-5 and ZnPC-pz with a statistical sequence of stacking modes with the ratio of stacking types determined by Maxwell-Boltzmann energy distribution. This allowed us to simulate PXRD patterns that closely match the experimental data, both in peak intensities and shapes. The rarely considered ABC stacking mode turns out to be crucial for LCOFs, being the most stable mode of COF-1 and ZnPc-pz. The presence of solvent molecule results in different LCOF structures, e.g. in assynthesized COF-1 with small pores. We have described an experimentally observed change of 
COF-1 stacking order accompanying solvent evacuation, which leads to structures with a mixed $\mathrm{AA}_{s} / \mathrm{AB}(\mathrm{C})$ stacking order. While we have only studied $h c b$ and $s q l$ LCOFs, we expect that our method is transferable to other layered framework materials including LCOFs and LMOFs.

\section{ASSOCIATED CONTENT}

Supporting Information. The Supporting Information is available free of charge on the ACS Publications website at DOI:

Details of the DFTB and DFT calculations, stacking modes and corresponding relative energies, the statistical model, more results of PXRD patterns and electronic properties.

\section{AUTHOR INFORMATION}

\section{Corresponding Author}

Thomas Heine - Technische Universität Dresden Theoretische Chemie Bergstraße 66c, 01062 Dresden, Germany; Helmholtz-Zentrum Dresden-Rossendorf, Institute of Resource Ecology, Leipzig Research Branch, Permoser Str. 15, 04316 Leipzig; Department of Chemistry, Yonsei University, Seodaemun-gu, Seoul 120-749, Korea; orcid.org/0000-0003-2379-6251; Email: thomas.heine@tu-dresden.de

Miroslav Položij - Technische Universität Dresden Theoretische Chemie Bergstraße 66c, 01062 Dresden, Germany; orcid.org/0000-0002-3016-9523; Email: miroslav.polozij@tu-dresden.de

\section{Authors}


Yingying Zhang - Technische Universität Dresden Theoretische Chemie Bergstraße 66c, 01062

Dresden, Germany; orcid.org/ 0000-0003-2135-3799; Email: Yingying.zhang@tu-dresden.de

\section{Author Contributions}

The manuscript was written through contributions of all authors. All authors have given approval to the final version of the manuscript.

\section{Funding Sources}

China Scholarship Council, Alexander-von Humboldt foundation, Deutsche Forschungsgemeinschaft (CRC 1415).

\section{Notes}

The authors declare no competing financial interest.

\section{ACKNOWLEDGMENT}

The authors gratefully acknowledge ZIH Dresden for computer time. Y.Z. acknowledges China Scholarship Council. M.P. acknowledges the Alexander-von-Humboldt Foundation for funding. All authors thank Deutsche Forschungsgemeinschaft for sup-port within CRC 1415.

\section{REFERENCES}

(1) Mahmood, J.; Ahmad, I.; Jung, M.; Seo, J.-M.; Yu, S.-Y.; Noh, H.-J.; Kim, Y. H.; Shin, H.J.; Baek, J.-B. Two-dimensional amine and hydroxy functionalized fused aromatic covalent organic framework. Commun. Chem. 2020, 3, 1-6. 
(2) Chen, X.; Geng, K.; Liu, R.; Tan, K. T.; Gong, Y.; Li, Z.; Tao, S.; Jiang, Q.; Jiang, D. Covalent Organic Frameworks: Chemical Approaches to Designer Structures and Built-In Functions. Angew. Chem. Int. Ed. Engl. 2020, 59, 5050-5091.

(3) Cui, D.; Perepichka, D. F.; MacLeod, J. M.; Rosei, F. Surface-confined single-layer covalent organic frameworks: design, synthesis and application. Chem. Soc. Rev. 2020, 49, 2020-2038.

(4) Lohse, M. S.; Bein, T. Covalent Organic Frameworks: Structures, Synthesis, and Applications. Adv. Funct. Mater. 2018, 28, 1705553.

(5) Haase, F.; Gottschling, K.; Stegbauer, L.; Germann, L. S.; Gutzler, R.; Duppel, V.; Vyas, V. S.; Kern, K.; Dinnebier, R. E.; Lotsch, B. V. Tuning the stacking behaviour of a 2D covalent organic framework through non-covalent interactions. Mater. Chem. Front. 2017, 1, 1354-1361.

(6) Hulliger, F.; Lévy, F. Structural Chemistry of Layer-Type Phases; Physics and Chemistry of Materials with Layered Structures, Vol. 5; Springer Netherlands, 1976.

(7) Zhang, Y.-B.; Su, J.; Furukawa, H.; Yun, Y.; Gándara, F.; Duong, A.; Zou, X.; Yaghi, O. M. Single-crystal structure of a covalent organic framework. J. Am. Chem. Soc. 2013, 135, 1633616339.

(8) Ma, T.; Kapustin, E. A.; Yin, S. X.; Liang, L.; Zhou, Z.; Niu, J.; Li, L.-H.; Wang, Y.; Su, J.; Li, J.; et al. Single-crystal x-ray diffraction structures of covalent organic frameworks. Science 2018, 361, 48-52.

(9) Lukose, B.; Kuc, A.; Heine, T. The structure of layered covalent-organic frameworks. Chemistry (Weinheim an der Bergstrasse, Germany) 2011, 17, 2388-2392. 
(10) Côté, A. P.; Benin, A. I.; Ockwig, N. W.; O'Keeffe, M.; Matzger, A. J.; Yaghi, O. M. Porous, crystalline, covalent organic frameworks. Science 2005, 310, 1166-1170.

(11) Dong, R.; Zhang, Z.; Tranca, D. C.; Zhou, S.; Wang, M.; Adler, P.; Liao, Z.; Liu, F.; Sun, Y.; Shi, W.; et al. A coronene-based semiconducting two-dimensional metal-organic framework with ferromagnetic behavior. Nat. Commun. 2018, 9, 2637.

(12) Pütz, A. M.; Terban, M. W.; Bette, S.; Haase, F.; Dinnebier, R. E.; Lotsch, B. V. Total scattering reveals the hidden stacking disorder in a 2D covalent organic framework. Chem. Sci. 2020, 11, 12647-12654.

(13) Položij, M.; Thang, H. V.; Rubeš, M.; Eliášová, P.; Čejka, J.; Nachtigall, P. Theoretical investigation of layered zeolites with MWW topology: MCM-22P vs. MCM-56. Dalton Trans. 2014, 43, 10443-10450.

(14) Teteruk, J. L.; Glinnemann, J.; Gorelik, T. E.; Linden, A.; Schmidt, M. U. Explanation of the stacking disorder in the $\beta$-phase of Pigment Red 170. Acta Cryst. B 2014, 70, 296-305.

(15) Wang, M.; Ballabio, M.; Wang, M.; Lin, H.-H.; Biswal, B. P.; Han, X.; Paasch, S.; Brunner, E.; Liu, P.; Chen, M.; et al. Unveiling Electronic Properties in Metal-Phthalocyanine-Based Pyrazine-Linked Conjugated Two-Dimensional Covalent Organic Frameworks. J. Am. Chem. Soc. 2019, 141, 16810-16816.

(16) Wu, X.; Han, X.; Liu, Y.; Liu, Y.; Cui, Y. Control Interlayer Stacking and Chemical Stability of Two-Dimensional Covalent Organic Frameworks via Steric Tuning. J. Am. Chem. Soc. 2018, 140, 16124-16133. 
(17) Schwarz, D.; Acharjya, A.; Ichangi, A.; Kochergin, Y. S.; Lyu, P.; Opanasenko, M. V.; Tarábek, J.; Vacek Chocholoušová, J.; Vacek, J.; Schmidt, J.; et al. Tuning the Porosity and Photocatalytic Performance of Triazine-Based Graphdiyne Polymers through Polymorphism. ChemSusChem 2019, 12, 194-199.

(18) Albacete, P.; Martínez, J. I.; Li, X.; López-Moreno, A.; Mena-Hernando, S. A.; PlateroPrats, A. E.; Montoro, C.; Loh, K. P.; Pérez, E. M.; Zamora, F. Layer-Stacking-Driven Fluorescence in a Two-Dimensional Imine-Linked Covalent Organic Framework. J. Am. Chem. Soc. 2018, 140, 12922-12929.

(19) Ghosh, S.; Nakada, A.; Springer, M. A.; Kawaguchi, T.; Suzuki, K.; Kaji, H.; Baburin, I.; Kuc, A.; Heine, T.; Suzuki, H.; et al. Identification of Prime Factors to Maximize the Photocatalytic Hydrogen Evolution of Covalent Organic Frameworks. J. Am. Chem. Soc. 2020, $142,9752-9762$.

(20) Kuc, A.; Springer, M. A.; Batra, K.; Juarez - Mosqueda, R.; Wöll, C.; Heine, T. Proximity Effect in Crystalline Framework Materials: Stacking - Induced Functionality in MOFs and COFs. Adv. Funct. Mater. 2020, 30, 1908004. 
Table of Content Graphic

Solvent molecules

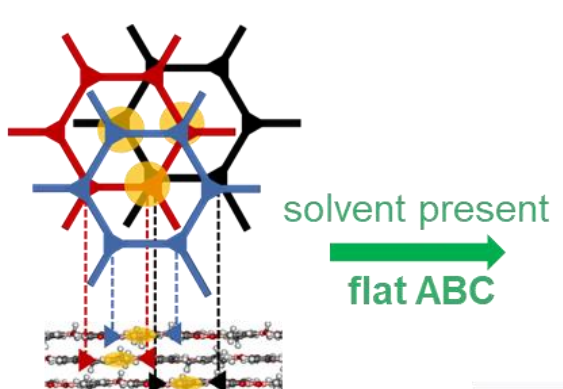

-60 of

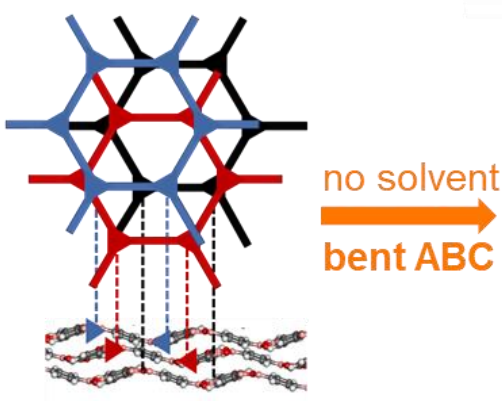

Real structure Lateral shift

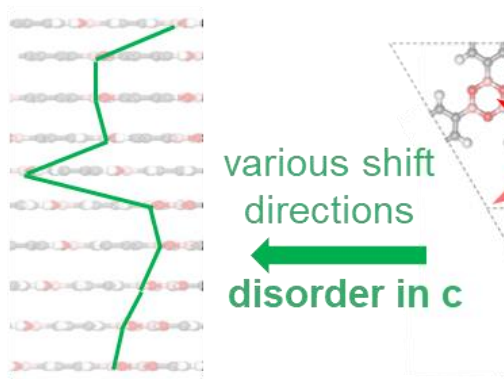

Idealistic model
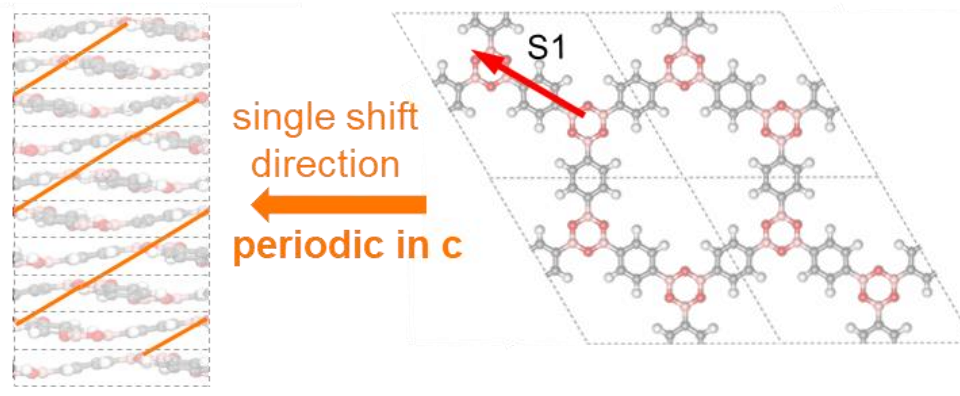